\title{
Numerical simulation of gas-bubble formation through two submerged orifices
}

\author{
V K PRASAD ${ }^{1}$, D CHATTERJEE ${ }^{2, *}$ and S P SINGH ${ }^{3}$ \\ ${ }^{1}$ Mobile Cargo Handling Equipment, Mechanical and Electrical Engineering, Kolkata Port Trust, \\ Kolkata 700001, India \\ ${ }^{2}$ Advanced Design and Analysis Group, CSIR-Central Mechanical Engineering Research Institute, \\ Durgapur 713209, India \\ ${ }^{3}$ Surface Engineering and Tribology Group, CSIR-Central Mechanical Engineering Research Institute, \\ Durgapur 713209, India \\ e-mail: d_chatterjee@cmeri.res.in
}

MS received 12 October 2017; revised 6 February 2018; accepted 23 April 2018; published online 12 September 2018

\begin{abstract}
In this work, two-dimensional numerical simulations are carried out to analyse the dynamics of gasbubble formation at two adjacent orifices submerged in an immiscible Newtonian liquid under the condition of constant gas flow rate using finite-volume-based commercial CFD solver Ansys Fluent. Two conditions for the ambient liquid are considered: firstly the liquid is in quiet condition and secondly, it is also co-flowing with the gas stream. The full cycle from the bubble formation to its detachment and the corresponding dynamics are simulated using the volume of fluid (VOF) method, which is one of the efficient front capturing techniques of Eulerian family. The study includes: (i) time sequence profile of bubble formation to clearly represent the bubble growth, neck formation and bubble break-up at a given Weber number $(W e)$, Reynolds number $(R e)$, Bond number $(B o)$ and liquid to gas mean velocity ratio $\left(V_{r}\right)$; (ii) bubble growth history for different $V_{r}$ and at constant $R e, W e$ and $B o$; (iii) effect of orifice spacing on bubbling synchronicity and frequency at given $V_{r}, W e, R e$ and $B o$ and (iv) synchronous bubbling regime, bubble size and formation time determination, bubble coalescence phenomenon and technique for its inhibition.
\end{abstract}

Keywords. Bubble dynamics; coalescence; VOF; bubbling frequency and synchronicity; submerged orifices.

\section{Introduction}

Gas bubbles have wide-spread applications in many processes involving stirring, mixing, water treatment, water oxygenation, etc. and are often encountered in metallurgical, chemical, nuclear and food industries [1]. Besides this, the small size gas bubbles also have potential applications in the field of medical science; for instance, micron-sized bubbles can be used as ultrasound contrast agents (UCAs), tumour destructors, thrombus destructors and genes/drug carriers. Micro-bubbles are specifically used as a drug carrier to deliver a therapeutic drug (encapsulated with the micro-bubble) at specific point of interest in the human body. The gas bubble, when used as an UCA and drug carrier, not only enhances the quality of ultrasound diagnostic images for better diagnosis but also improves the effectiveness of the treatment by facilitating the sitespecific delivery of the drug [2].

A most general way of generating gas bubbles in a fluid medium is to inject a gas continuously through one or more

*For correspondence orifices, which emanates and disperses into the quiescent or co-flowing liquid medium and breaks up into bubbles. In co-flow configuration, the dispersed phase (gas) is injected through a nozzle or orifice in the direction of liquid flow whereas in counter-flow configuration, the dispersed phase is introduced in the direction exactly opposite to that of the liquid flow. However, in cross-flow configuration, the dispersed phase is introduced in the direction perpendicular to that of the liquid flow $[3,4]$. Some major advantages of the co-flowing liquid medium over the quiescent medium are: (i) less probability of bubbles merger/coalescence; (ii) generation of smaller size bubbles and (iii) enhanced bubble formation frequency and reduced bubble formation time.

The numerical simulation of dynamics of gas-liquid involves the solution of continuity and momentum equations for the two fluids involving the stress balance equation at the interface. The depiction of interface offers a particular challenge that can be dealt numerically by VOF (volume of fluid) method [5, 6] in association with PLIC (Piecewise Linear Interface Calculation) algorithm [7]. This method has the advantage of accurately solving the 
interface advection equation without compromising the mass balance but it lacks the capability to calculate excellent geometrical properties such as normals and/or curvatures. However, with the implementation of the PLIC algorithm to construct the evolving interface using the VOF function this issue can be addressed. The PLIC algorithm consists of two steps, namely propagation/advection step and reconstruction step.

To understand the underlying physics, the bubble formation at a single orifice submerged in a liquid pool (either quiescent or co-flowing) has been studied largely as a basic problem despite knowing the fact that it is not in wide use practically. Bubble formation at a single submerged orifice has been studied by many researchers [8-14], which dealt with bubble formation in quiescent and co-flowing liquid ambience conditions. Chuang and Goldschmidt [15] were the first to propose a one-stage model for the bubble formation at a nozzle submerged in a co-flowing liquid. Their model was based on the force balance at bubble detachment. Sada et al [16] observed bubble formation at a single nozzle in water flowing parallel to the nozzle and observed the effect of liquid velocity on bubble detachment. Gerlach et al [17] applied a coupled level-set and volume-of-fluid (CLSVOF) [18] method to study the numerical simulation of bubble formation at a submerged orifice at constant inflow conditions and they individually examined the influences of gas flow rates, contact angle and fluid properties. Sevilla et al [19] performed a comprehensive experimental study on the periodic bubble formation from an air-injection needle in an air-water co-flowing system and also presented a model to describe the process under conditions of negligible buoyancy and viscous effects. Terasaka et al [20] experimentally investigated the effects of liquid velocity, nozzle diameter, gas chamber and gas flow rate on the volumes, shapes and growth rates of bubbles formed at a nozzle submerged in a co-flowing liquid. Gordillo et al [21] studied the mechanisms of the bubble formation from a needle in a co-flowing liquid at high $R e$ and high We numbers with the aid of experiments and boundary-integral numerical simulations. Chakraborty et al [22] studied the differences between the dynamic effects of quiescent and co-flowing liquids on bubble evolution and break-up processes for a constant gas flux injected from a single submerged orifice.

On the contrary, only few studies have been performed on the bubble formation at multiple orifices, leading to a dearth of information on how the growing bubbles evolve and interact with each other at two adjacent orifices in bubble columns. Also, there is a lack of fundamental understanding in correlating the comprehensive body of knowledge gained from single-orifice study with industrial multi-orifice problems. Zuiderweg [23], Wijn [24], Miyahara et al [25] and Titomanlio et al [26] carried out experimental investigations on bubble formation over an industrial perforated plate to investigate size of the bubbles generated and different types of flow regimes. Ruzicka et al
[27, 28] experimentally investigated the formation of gas bubbles at two orifices and identified two typical modes of bubbling: the synchronous and the asynchronous, and concluded that spacing between the orifices plays a key role in the mechanisms of bubbling interactions. Xie and Reginald [29] investigated gas-bubble formation at two and multiple orifices and identified three modes of bubbling regimes (synchronous, alternate and unsteady) depending on the gas flow rates and orifice spacing. Pereira Dias [30] found experimentally that the size, frequency and formation process of bubbles at multiple orifices are influenced by the distance between the orifices as it plays a critical role on the coalescence phenomenon of bubbles growing at neighbouring orifices.

It is therefore crucial to say that more work is needed to understand the behaviour of bubble formation in a two submerged orifice system involving constant rate of gas injection in either quiescent or co-flowing liquid ambience. In this work, we attempt to analyse numerically the bubble formation phenomenon at two submerged orifices using the VOF method and also to elucidate the underlying physics of bubble dynamics governed by the interplay of inertial, pressure, surface tension and buoyancy forces. The work also presents a systematic study of bubble formation, bubbling synchronicity and frequency at the two adjacent orifices.

\section{Formulation of the problem}

A two-dimensional co-flowing arrangement of two immiscible fluids is shown in figure 1. A gas of viscosity $\mu_{g}$ and density $\rho_{g}$ is injected at a constant flow rate $Q_{g}$ with a corresponding mean velocity $v_{d, a v g}$ through the two orifices (certain distance apart) with the orifice radius $r_{2}$ each into a Newtonian liquid ambient of viscosity $\mu_{l}$ and density $\rho_{l}$ in a tank of width $2 R_{3}$ (with $R_{3}$ being the distance of vertical wall from the central line $=3 r_{1}+2 r_{2}$ taken in such a fashion that the friction effect of wall on bubble growth can be neglected). The surface tension coefficient $\sigma$ at the gasliquid interface is assumed to be spatially uniform and constant in time. The gravitational force acts along the central line in downward direction. The co-flowing liquid flows in upward direction in the tank with an average velocity $v_{c, a v g}$. A two-dimensional coordinate system $(r, z)$ is defined whose origin lies on the central line, where $r$ represents the horizontal coordinate and $z$ is the vertical coordinate measured in the opposite direction of gravity. The height of the tank is taken as $H_{t}\left(=60 r_{2}\right)$ to ensure that dynamics of bubble formation is not influenced by the outflow boundary condition at the outlet.

Here, the submerged orifices are assumed to have negligible thickness for simplicity. Additionally, two more significant simplifications are made: firstly, the bubbling at the two orifices is synchronous (i.e., in phase) and secondly, the side-by-side interaction of adjacent bubbles is not included in the modelling. 


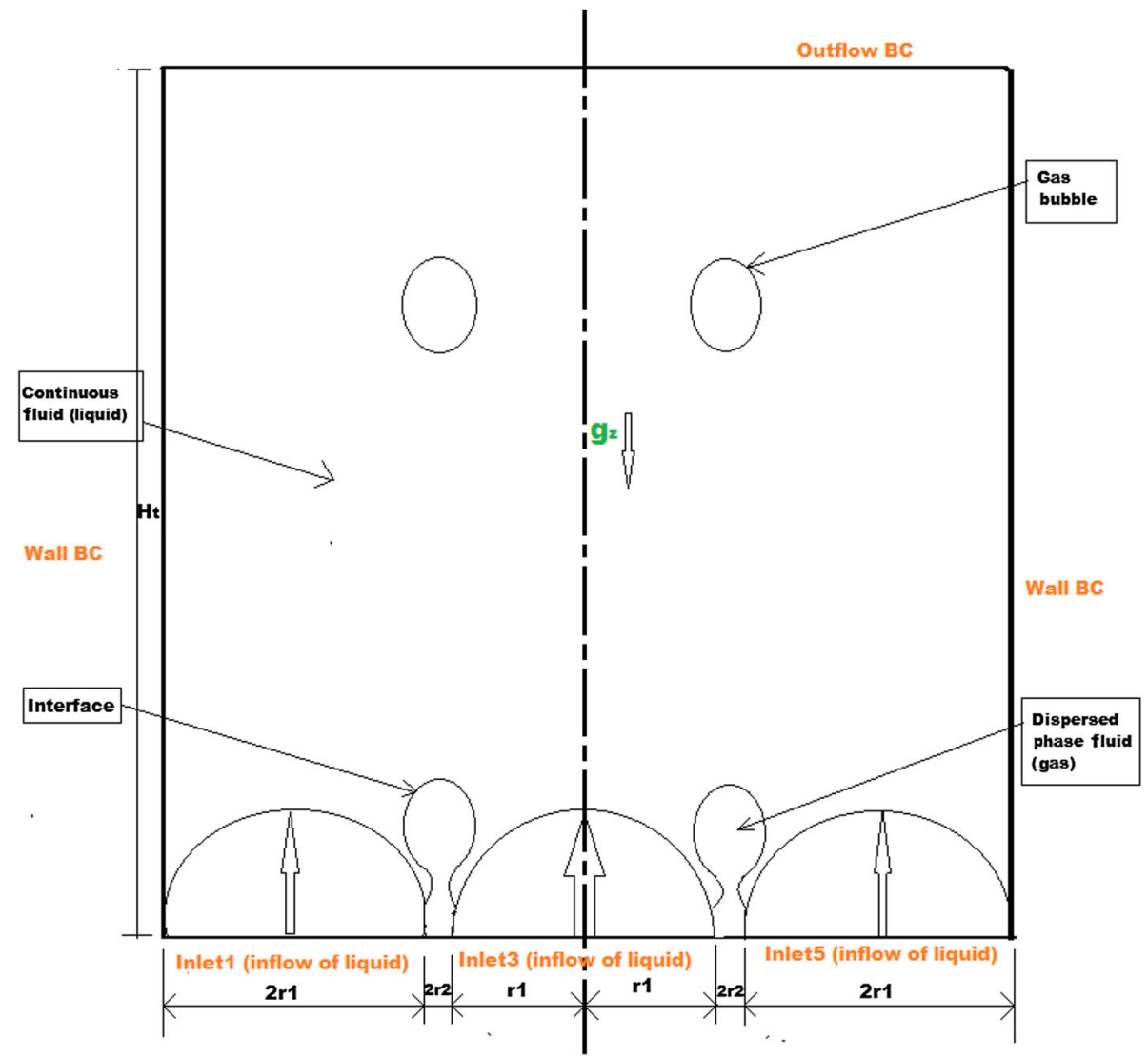

Figure 1. Computational domain and imposed boundary conditions on a two-dimensional (cylindrical) plane. Origin is at the middle point of the bottom boundary.

\subsection{Governing equations}

The mass and momentum conservation equations for the incompressible Newtonian fluids are given as follows:

$$
\nabla \cdot \vec{V}=0,
$$

$\rho\left(\frac{\partial \vec{V}}{\partial t}+\nabla \cdot \vec{V} \vec{V}\right)=-\nabla P+\rho \vec{g}+\nabla \cdot\left[\mu\left(\nabla \vec{V}+(\nabla \vec{V})^{T}\right)\right]$.

Since we have considered two incompressible and immiscible Newtonian fluids, there will be an interface separating these two phases. Thus, the modified momentum equation, which includes the surface tension force at the interface, can be given as

$$
\begin{aligned}
\rho\left(\frac{\partial \vec{V}}{\partial t}+\nabla \cdot \vec{V} \vec{V}\right)= & -\nabla P+\rho \vec{g}+\nabla \cdot\left[\mu\left(\nabla \vec{V}+(\nabla \vec{V})^{T}\right)\right] \\
& +\sigma \kappa \vec{n} \delta_{s} .
\end{aligned}
$$

Hence, the momentum equation using the concept of Brackbill et al [31] can now be written as

$$
\begin{aligned}
\rho\left(\frac{\partial \vec{V}}{\partial t}+\nabla \cdot \vec{V} \vec{V}\right)= & -\nabla P+\rho \vec{g}+\nabla \cdot\left[\mu\left(\nabla \vec{V}+(\nabla \vec{V})^{T}\right)\right] \\
& +\sigma \kappa \nabla \alpha
\end{aligned}
$$

where $\alpha$ is the void fraction of liquid in a two-phase cell.

The governing equations can be non-dimensionalized using the following schemes:

$$
\begin{aligned}
& z^{*}=\frac{z}{r_{2}}, r^{*}=\frac{r}{r_{2}}, V^{*}=\frac{V}{v_{d, a v g}}, \\
& t^{*}=\frac{t \times v_{d, a v g}}{r_{2}}, p^{*}=\frac{p}{\rho_{l}\left(v_{d, a v g}^{2}\right)}, \\
& \rho^{*}=\frac{\rho}{\rho_{l}}, \mu^{*}=\frac{\mu}{\mu_{l}}, K^{*}=\frac{\kappa}{r_{2}^{-1}}
\end{aligned}
$$

where $r_{2}$ and $v_{d, a v g}$ are the characteristic length and velocity, respectively, the subscript $l$ represents liquid and the superscript $*$ represents the dimensionless quantities. Using the non-dimensionalization schemes described in Eq. (5), Eqs. (1) and (4) assume following dimensionless forms: 


$$
\begin{aligned}
\nabla & \cdot \vec{V}^{*}=0 \\
\rho^{*}\left(\frac{\partial \vec{V}^{*}}{\partial t^{*}}+\nabla \cdot \vec{V}^{*} \vec{V}^{*}\right)= & -\nabla p *+\rho^{*}\left(\frac{B o}{W e}\right) \\
& +\left(\frac{1}{\operatorname{Re}}\right) \nabla \cdot\left[\nabla \vec{V}^{*}+\left(\nabla \vec{V}^{*}\right)^{T}\right] \\
& +\left(\frac{1}{W e}\right) K^{*} \nabla \alpha
\end{aligned}
$$

where $B o=\rho_{1} g r_{2}^{2} / \sigma, W e=\rho_{1} v_{d, a v g}^{2} r_{2} / \sigma, R e=\rho_{1} v_{d, a v g} r_{2} /$ $\mu_{l}$ are three different dimensionless parameters, which govern the multiphase flow phenomena.

The viscosity and density across the interface are interpolated linearly using the void fraction field as follows:

$$
\begin{aligned}
& \rho(\alpha)=\rho_{g}+\alpha\left(\rho_{l}-\rho_{g}\right), \\
& \mu(\alpha)=\mu_{g}+\alpha\left(\mu_{l}-\mu_{g}\right) .
\end{aligned}
$$

In order to track the instantaneous position of the interface using volume fraction function $(\alpha)$, advection/transport equation for $\alpha$ is solved, which is given as

$$
\frac{\partial \alpha}{\partial t}+\vec{V} \cdot(\nabla \alpha)=0
$$

At each time step, the algorithm first calculates the flow field by solving the continuity and momentum equations; then the advection equation (10) for $\alpha$ is solved in order to update the value of $\alpha$ in each cell. Thereafter, cell interface is reconstructed using the PLIC method [7] that is either parallel to the cell wall [32] or sloped, using the values of $\alpha$ of the surrounding cells. The position of the line inside the cell is such that the volume fraction of liquid is equal to $\alpha$, and boundary conditions are imposed on the interface.

\subsection{Boundary conditions}

\section{Inlet $B C(z=0)$}

A fully developed Poiseuille flow is imposed at the orifice inlet:

$$
\begin{aligned}
& \begin{array}{l}
u_{d}^{*}(r)=0 \\
v_{d}^{*}(r)=\left(\frac{1}{r_{2}^{2}}\right) *\left[-r^{2}+2 *( \pm r) * R_{1}-\left(r_{1}^{2}+2 * r_{1} * r_{2}\right)\right]
\end{array} \\
& \quad \alpha=0, z=0 \\
& \text { where } R_{1}=\left(r_{1}+r_{2}\right), \\
& \quad R_{2}=\left(r_{1}+2 r_{2}\right) \\
& \text { for }-R_{2} \leq r \leq-r_{1}: \text { negative sign will be considered. } \\
& \qquad r_{1} \leq r \leq R_{2}: \text { positive sign will be considered. }
\end{aligned}
$$

where $u_{d}^{*}$ and $v_{d}^{*}$ are the non-dimensional horizontal and vertical components of the velocity of the gas phase, respectively, in their dimensionless forms. They are non-dimensionalized using the average velocity of dispersed/gas phase $v_{d, a v g}$.

Similarly, a fully developed Poiseuille flow is imposed at the inlet point of the ambient liquid (continuous phase) immediate to the side wall:

$u_{c w}^{*}(r)=0$,

$v_{c w}^{*}(r)=2 * V_{r} *\left[\frac{1-\left(\frac{r}{R_{3}}\right)^{2}+\frac{1-\left(\frac{R_{2}}{R_{3}}\right)^{2}}{\ln \left(\frac{R_{3}}{R_{2}}\right)} \log \left(\frac{ \pm r}{R_{3}}\right)}{1+\left(\frac{R_{2}}{R_{3}}\right)^{2}-\frac{1-\left(\frac{R_{2}}{R_{3}}\right)^{2}}{\log \left(\frac{R_{3}}{R_{2}}\right)}}\right] ;$

$\alpha=1, z=0$

where $R_{2}=\left(r_{1}+2 r_{2}\right)$ and $R_{3}=\left(3 r_{1}+2 r_{2}\right)$

for $-R_{3} \leq r \leq-R_{2}$ : negative sign will be considered.

$R_{2} \leq r \leq R_{3}$ : positive sign will be considered.

where $u_{c w}^{*}$ and $v_{c w}^{*}$ are the non-dimensional horizontal and vertical components of velocity, respectively, of the continuous liquid near the side wall and $V_{r}\left(=v_{c, \text { avg }} / v_{d, \text { avg }}\right)$ is the liquid to gas mean velocity ratio, which actually takes into account the dynamic effect of co-flowing liquid.

In the same way a fully developed Poiseuille flow is imposed on the inlet point of the ambient liquid lying between the two adjacent orifices:

$$
\begin{aligned}
& u_{c o}^{*}(r)=0, \\
& v_{c o}^{*}(r)=2 v_{c, \text { avg }}\left[1-\left(\frac{r}{r_{1}}\right)^{2}\right] \\
& \text { at } \alpha=1, z^{*}=0, \\
& \text { for }-r_{1} \leq r \leq+r_{1}
\end{aligned}
$$

Here, $u_{c o}^{\prime}$ and $v_{c o}^{\prime}$ are the horizontal and vertical components of the velocity of the continuous liquid between the two orifices, respectively.

Outlet $B C\left(z=H_{t}\right)$

At the top edge of the computational domain, an outflow boundary condition is imposed, which essentially means that there is no change in the properties along the vertical direction at outlet point:

$$
\begin{aligned}
& \frac{\partial u^{*}}{\partial z^{*}}=\frac{\partial v^{*}}{\partial z^{*}}=\frac{\partial \alpha}{\partial z^{*}}=0 \\
& \text { at } z=H_{t}, \text { for }-R_{3} \leq r \leq+R_{3} \\
& P=P_{0}\left(\rho_{l} v_{d, a v g}^{2}\right)
\end{aligned}
$$$$
\text { where } P_{0}=\text { atmospheric pressure }
$$ 
Wall $B C\left(r= \pm R_{t}\right)$

At the wall of the tank, no slip and no penetration boundary conditions are applied:

$$
u=v=0, \text { at } r= \pm R_{3}, \text { for } 0 \leq z \leq H_{t} .
$$

\section{Initial conditions}

The bubbles are initially assumed to be of hemispherical (semicircular in 2D space) shape of radius equal to the orifice radius:

$$
(r)^{2}+(z)^{2}=\left(r_{2}\right)^{2} .
$$

Both the disperse and continuous phases are assumed to be initially quiescent and at uniform pressure:

$$
\begin{aligned}
& u(r, z ; t=0)=0, \\
& v(r, z ; t=0)=0, \\
& P(r, z ; t=0)=\text { constant }
\end{aligned}
$$

\subsection{Solution methodologies}

The commercial CFD solver Ansys Fluent [33] is used for the numerical computation of the problem. The VOF multiphase model of Ansys is deployed. The QUICK (Quadratic Upstream Interpolation Convective Kinetics) scheme [34] is used for the discretization of the convective terms in the momentum equation. The Geometric reconstruction scheme is used for the interface reconstruction and the PRESTO (PREssure STaggering Option) scheme [35] is used for the pressure discretization. Fractional step scheme is used for the pressurevelocity coupling. An implicit first-order time discretization scheme is used and a sufficiently small dimensionless time step size, $10^{-5}$, is chosen. The time step size is so chosen that it satisfies the CFL (CourantFrederick-Levy) criterion [36]. According to the CFL criterion, the Courant number $(C)$ must satisfy the following inequality:

$$
C=\frac{u \Delta t}{\Delta r}+\frac{v \Delta t}{\Delta z} \leq C_{\max }
$$

where $u, v$ are the horizontal and vertical velocity components, respectively, $\Delta r$ and $\Delta z$ are the mesh sizes along two respective directions and $\Delta t$ is the time step size. The value of $C_{\max }$ changes according to the method used for solving the discretized equation, especially depending on whether the method is explicit or implicit. For an explicit scheme, typically $C_{\max }=1$. The implicit scheme is usually less sensitive to numerical instability and so larger values of $C_{\max }$ may be tolerated. The simulation starts with a low global Courant number such as $C=3$ with a chosen dimensionless time step size of $10^{-3}$. However, it increases gradually with simulation time and reaches around 250, which makes the solution unstable. The time step size is then reduced first to $10^{-4}$; the Courant number reduces initially and then again increases with simulation time and the solution once again becomes unstable. Then the time step size is further reduced to $10^{-5}$, which makes the solution stable.

In the VOF method, a single set of conservation equations is shared by both the phases and the volume fraction of each of the phases is tracked in each computational cell throughout the domain [5]. For each additional phase that is added to the model, a variable is introduced: the volume fraction $(\alpha)$ of the phase in the computational cell. In each control volume, the volume fractions of all phases sum up to unity. All control volumes must be filled with either a single-phase fluid or a combination of fluids of different phases as the VOF method does not allow for any void regions in the domain. It must be noted here that the phase volume fraction values do not uniquely identify the interface and different interface configurations may correspond to the same values of volume fractions; hence in order to track the geometry of the interface accurately, PLIC algorithm [7] is employed.

A uniform grid structure is used to discretize the entire computational domain. The smallest division along both horizontal and vertical directions is taken to be $0.1525 \mathrm{~mm}$. A comprehensive grid sensitivity study is also conducted using three different grid sizes $(290 \times 300)$, $(390 \times 400)$ and $(580 \times 600)$. Computations are performed for $W e=$ 4.35, $R e=690.48$ and $B o=0.31$ and the evolution of the bubble is shown in figure 2 for various time instants. Figure 2 depicts the bubble shapes for all the three meshes $(290 \times 300),(390 \times 400)$ and $(580 \times 600)$ and they do not show significant differences among themselves. Besides this, a closer look at the figures reveals that diffusion of the interface in case of coarse grid has taken place over a thicker region as compared with diffusion of the interface in case of fine grid meshes (where the interface is diffused over a very thin region) under the same set of other parameters such as $V_{r}, W e, B o, R e$, etc. This essentially means that the interface is sharper in case of fine grid points. Hence, for all further studies, computations are performed on $(580 \times 600)$ grid points without compromising the accuracy of the numerical results. The following input parameters are used for the numerical computation to partially mimic the actual bubble formation process:

$$
\begin{aligned}
r_{2} & =1.525 \times 10^{-3} \mathrm{~m}, v_{d, a v g}=0.456 \mathrm{~ms}^{-1}, W e=4.35, R e \\
& =690.48, B o=0.31 .
\end{aligned}
$$

The physical properties of the fluids are given in table 1 .

\subsection{Validation}

The present numerical method is validated with the experimental results of Sada et al [16] and the numerical 


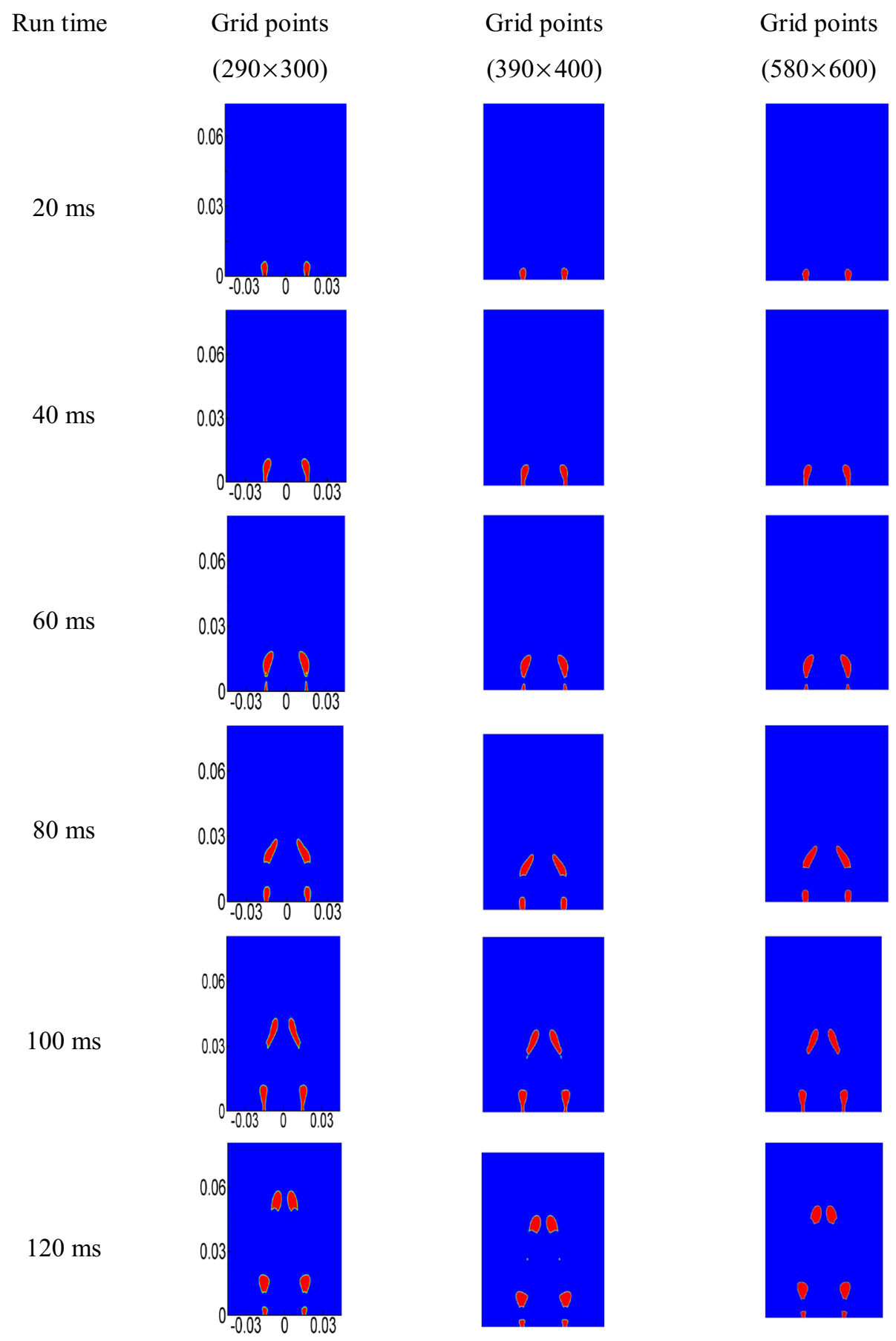

Figure 2. Grid independent test for three different mesh sizes $(290 \times 300),(390 \times 400)$ and $(580 \times 600)$ for $V_{r}=0.88$ at given $W e=4.35, R e=690.48$ and $B o=0.31$.

findings of Chakraborty et al [22] for the dynamic bubble formation from a single submerged orifice in an immiscible Newtonian liquid under the condition of constant gas inflow. Sada et al [16] conducted experiments with a cylindrical column of $5 \mathrm{~cm}$ diameter and $100 \mathrm{~cm}$ height in an infinite medium condition (negligible bounding wall effects) and Chakraborty et al [22] simulated the bubble formation numerically using a CLSVOF method. In the absence of sufficient experimental data for the case of two submerged orifices, the validation is performed for a single submerged orifice. Figure 3 shows a comparison in terms of the detached bubble diameter, which is computed as $D_{b}=$ $\sqrt[3]{6 V_{b} / \pi}$ where $V_{b}\left(\mathrm{~m}^{3}\right)$ is the bubble volume for a coflowing ambient liquid system. The pertinent geometric and controlling input parameters for the air-water system under consideration can be obtained from Chakraborty et al [22] 
Table 1. Physical properties of the reference fluids: air-water system at $20^{\circ} \mathrm{C}$.

\begin{tabular}{lc}
\hline Property & Value \\
\hline$\rho_{l}$ & $998.12 \mathrm{~kg} / \mathrm{m}^{3}$ \\
$\rho_{g}$ & $1.188 \mathrm{~kg} / \mathrm{m}^{3}$ \\
$\mu_{l}$ & $1.0016 \times 10^{-3} \mathrm{~Pa} \mathrm{~s}$ \\
$\mu_{g}$ & $1.824 \times 10^{-5} \mathrm{~Pa} \mathrm{~s}$ \\
$\sigma_{\mathrm{lg}}$ & $72.8 \times 10^{-3} \mathrm{~N} / \mathrm{m}$ \\
\hline
\end{tabular}

with the physical properties as given in table 1 . The comparison shows satisfactory agreement although certain discrepancy with the experimental results at low liquid mean velocity can be observed, which is attributed to the different procedure of calculating the bubble diameter adopted in the experimental study.

A further qualitative comparison is shown here for the bubble formation from two submerged orifices in figure 4 . A comparison is made to the experimental work of Xie and Reginald [29], where the experiments were carried out for air-water system from two orifices for quiet flow at $W e, R e$ and $B o$ numbers 4.2, 494.3 and 0.09, respectively. Their investigation was performed through high-speed photography and analysis of chamber pressure fluctuations. In the present case, values of $W e, R e$ and $B o$ are 4.35, 690 and 0.31 , respectively. Though these values do not match with the experimental work, their values are not very (or drastically) different. Figure 4 shows a qualitative comparison of the bubble formation to the experiment mentioned earlier and the present numerical computation at $V_{r}=0$, $t=80 \mathrm{~ms}$. The comparison shows good qualitative matching.

\section{Results and discussion}

We know that formation, expansion, neck formation and finally detachment of a gas bubble at the orifice tip is governed by the interplay of a number of forces such as the buoyancy force, pressure force, gas momentum force, surface tension force, liquid inertia force and drag force or fluid viscosity force. In the case of quiescent liquid ambience, the first three forces behave as detaching forces whereas the last three forces behave as attaching forces. However, in case of co-flowing liquid ambience, the liquid inertia force may act as either an attaching or a detaching force depending on the relative magnitude of the velocity of the co-flowing liquid and the bubble. If the bubble velocity is higher than the liquid mean velocity, then the liquid inertia force will act as opposing force (attaching force) and vice-versa.

Figure 5 describes the qualitative features of the bubble evolution with time for $W e=4.35, R e=690.48, B o=$

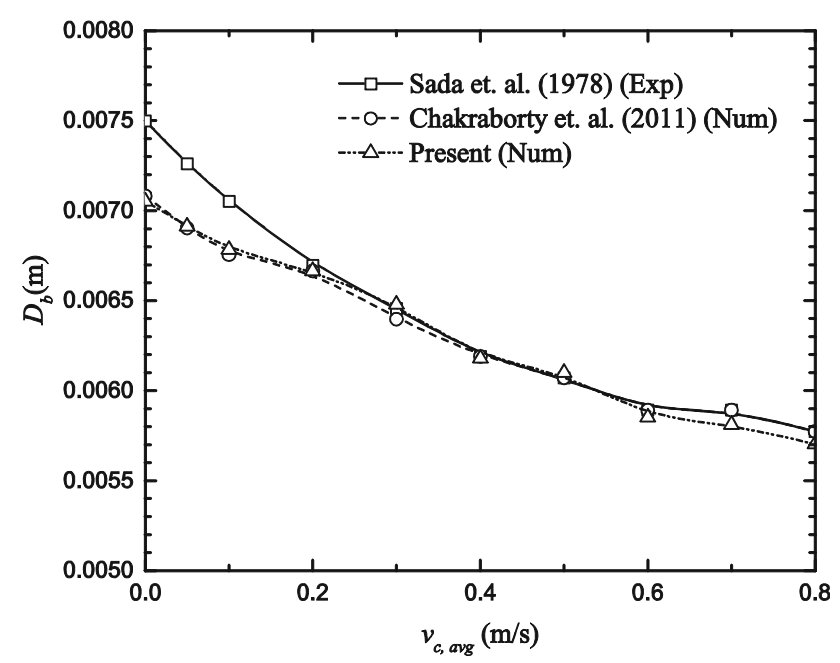

Figure 3. Variation of bubble diameter with liquid mean velocity evolved from two orifices in an air-water system for co-flowing ambient liquid system.

0.31 and $V_{r}=0.88$. Snapshots are shown in the increasing order of run time and the first figure represents the velocity profile imposed at different inlets for the two phases. After having a close look on the time sequence profiles, the bubble formation process can be divided into two stages: expansion stage and collapse stage. In the expansion stage, the total upward (detaching) force is in balance with the total downward (attaching) force. Now, any imbalance in the attaching and detaching forces will cause the commencement of collapse stage, which will ultimately lead to the formation of a new bubble. In the collapse stage, a visible neck forms (as depicted in figure 5 at $50 \mathrm{~ms}$ ) which goes on decreasing as the time elapses. In this stage, the coflowing liquid is assumed to have two components of velocity near the gas thread. The predominant horizontal velocity component perpendicular to the direction of the gas stream seems to compress the gas thread and forms the neck whereas the vertical component of the velocity elongates the gas thread in the axial direction. The lower part of the surface of the detached bubble is concave towards the liquid and the reason for this concavity is the retraction of the surface immediately after the bubble break-up. It is also observed that bubbles are approximately of hemispherical (semicircular in 2D) shape at lower heights; however, as they move downstream they tend to get spherical shape. It is further observed that the two adjacent bubbles try to diverge from the axis of the tank as they attend higher altitude.

Figure 6 (first row) shows the profile of the velocity vector at different inlet points with increasing liquid phase velocity as we move from left to right direction (since $V_{r}$ is increasing in this direction). Now, exactly similar figures in the second row ensure identical initial boundary condition imposed at the two orifices, with the assumption that the bubbles are initially hemispherical (semicircular in 2D) of 


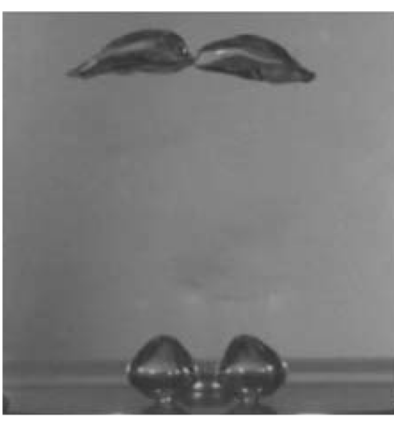

Xie and reginald

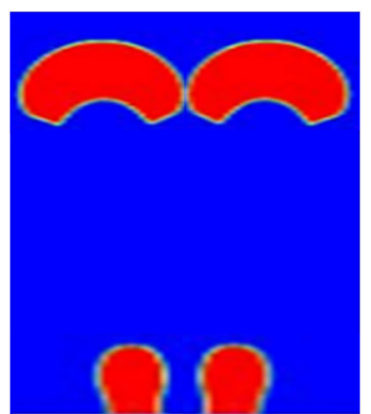

Present
Figure 4. Qualitative comparison of the bubble formation from two submerged orifices $\left(V_{r}=0, t=80 \mathrm{~ms}\right)$. radii equal to the orifice radius $\left(r_{2}\right)$. Here, the first column represents the case of quiescent liquid ambience; on the other hand, the second, third and fourth columns represent the case of co-flowing liquid ambience.

The main advantage of co-flowing liquid stream configuration over a quiescent liquid bath on bubble formation is that we can control the bubble size. In quiescent liquid, the inertia of the ambient liquid, caused by the expansion of an attached bubble at the orifice, exerts a downward force on the bubble surface and thereby acts as an opposing force to the bubble detachment. However, in the case of the co-flowing liquid ambience, the liquid inertia can be either attaching force or detaching force depending on the relative magnitude of the velocity of bubble and liquid mean velocity. When the bubble velocity is higher than that of the liquid, liquid inertia prevents bubble detachment and the liquid inertia term

Velocity

$t=0 \mathrm{~ms}$

$t=10 \mathrm{~ms}$

$t=20 \mathrm{~ms}$

$t=30 \mathrm{~ms}$

vector at inlet

$\mathrm{BC}$
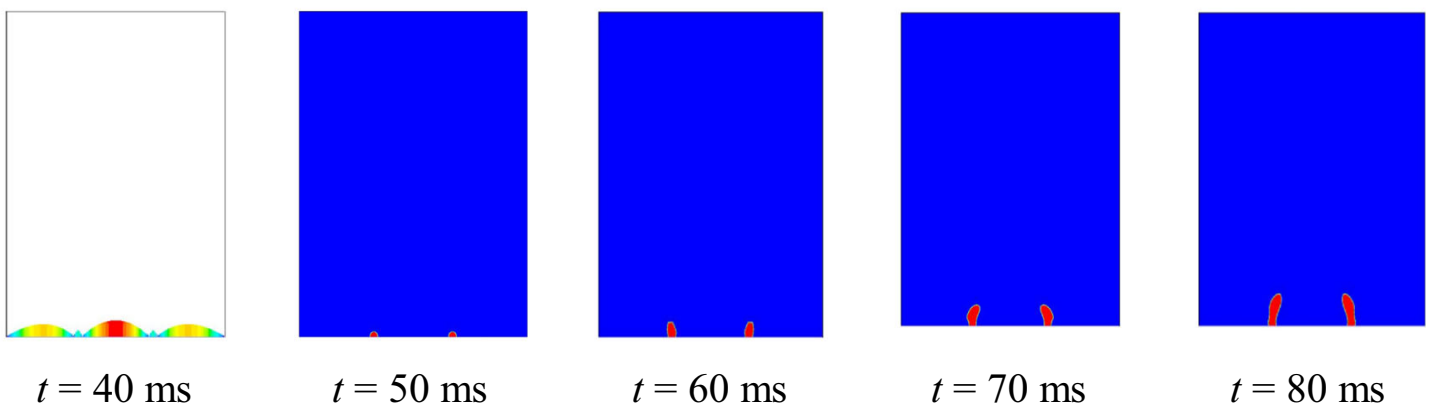

$$
t=80 \mathrm{~ms}
$$
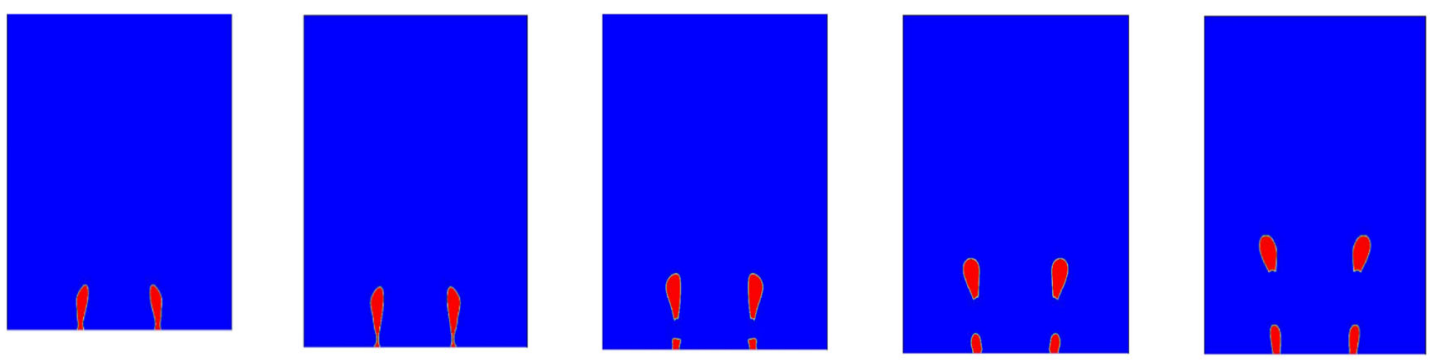

$$
t=90 \mathrm{~ms}
$$

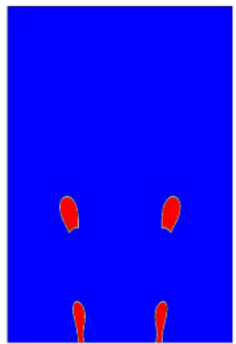

$$
t=100 \mathrm{~ms}
$$
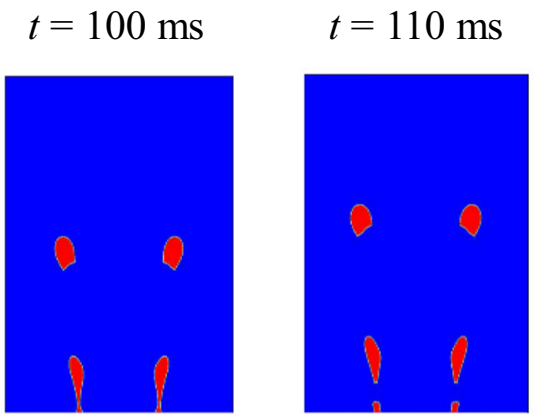

$t=120 \mathrm{~ms}$

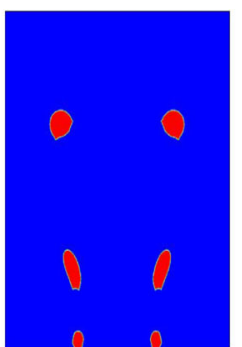

Figure 5. Time sequence profile of bubble growth, neck formation and bubble break-up for $W e=4.35, R e=690.48, B o=0.31$ and $V_{r}=1.32$. 


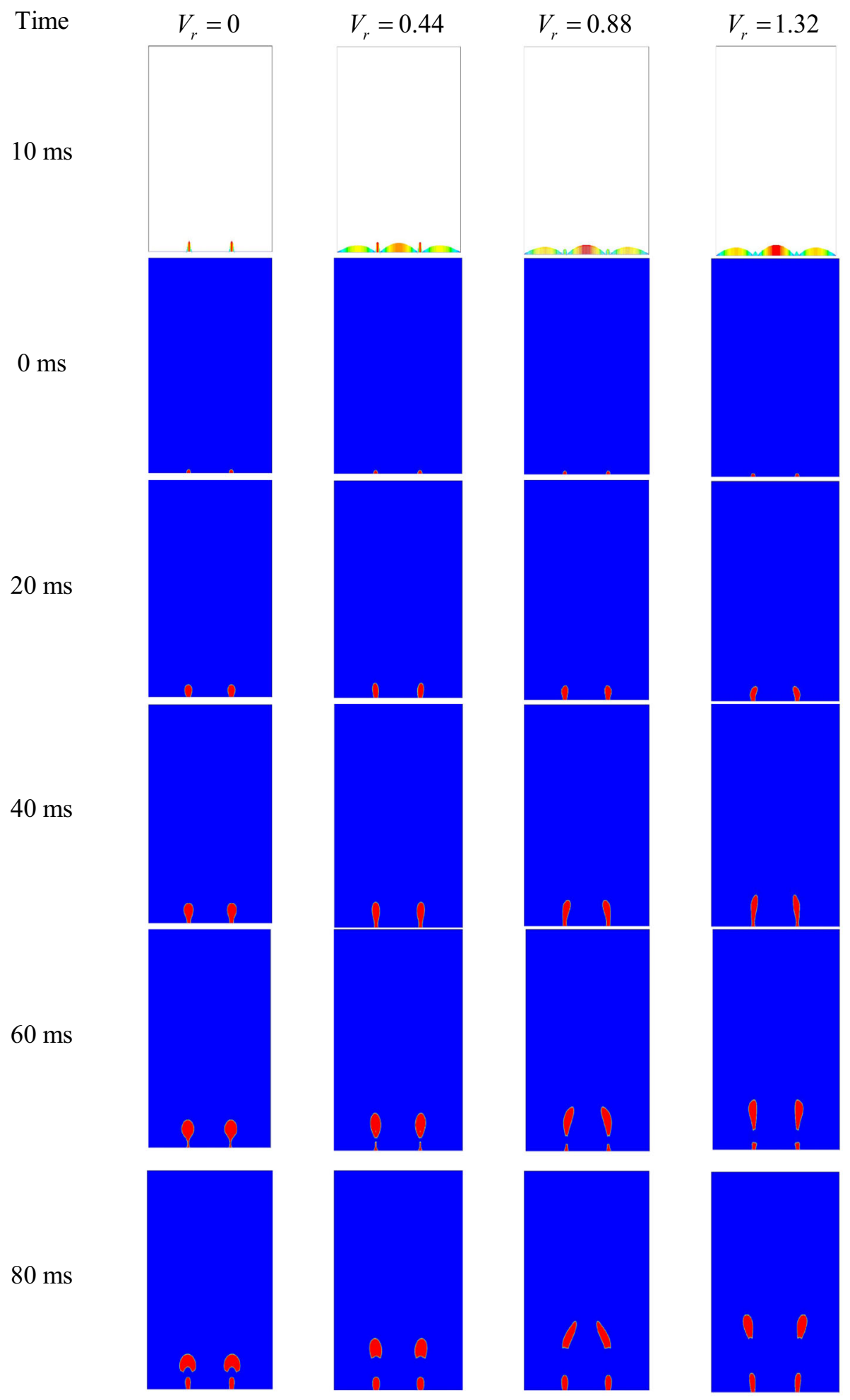

Figure 6. Effect of $V_{r}$ (liquid-to-gas mean velocity ratio) for $V_{r}=0,0.44,0.88,1.32$ at given $W e=4.35, R e=690.48$ and $B o=0.31$.

adds with the surface tension to oppose bubble detachment. However, when the liquid velocity is higher than the bubble, the liquid inertia favours bubble detachment and the liquid inertia term adds with the buoyancy force to drive the bubble detachment.
It is extremely important to note here that though the co-flowing configuration leads to an early detachment of forming bubbles and hence bubbles of small volume/size are formed in this case, yet the bubble growth rate $d V_{b} / d t$ remains unaffected by $V_{r}$, which is depicted by the almost 


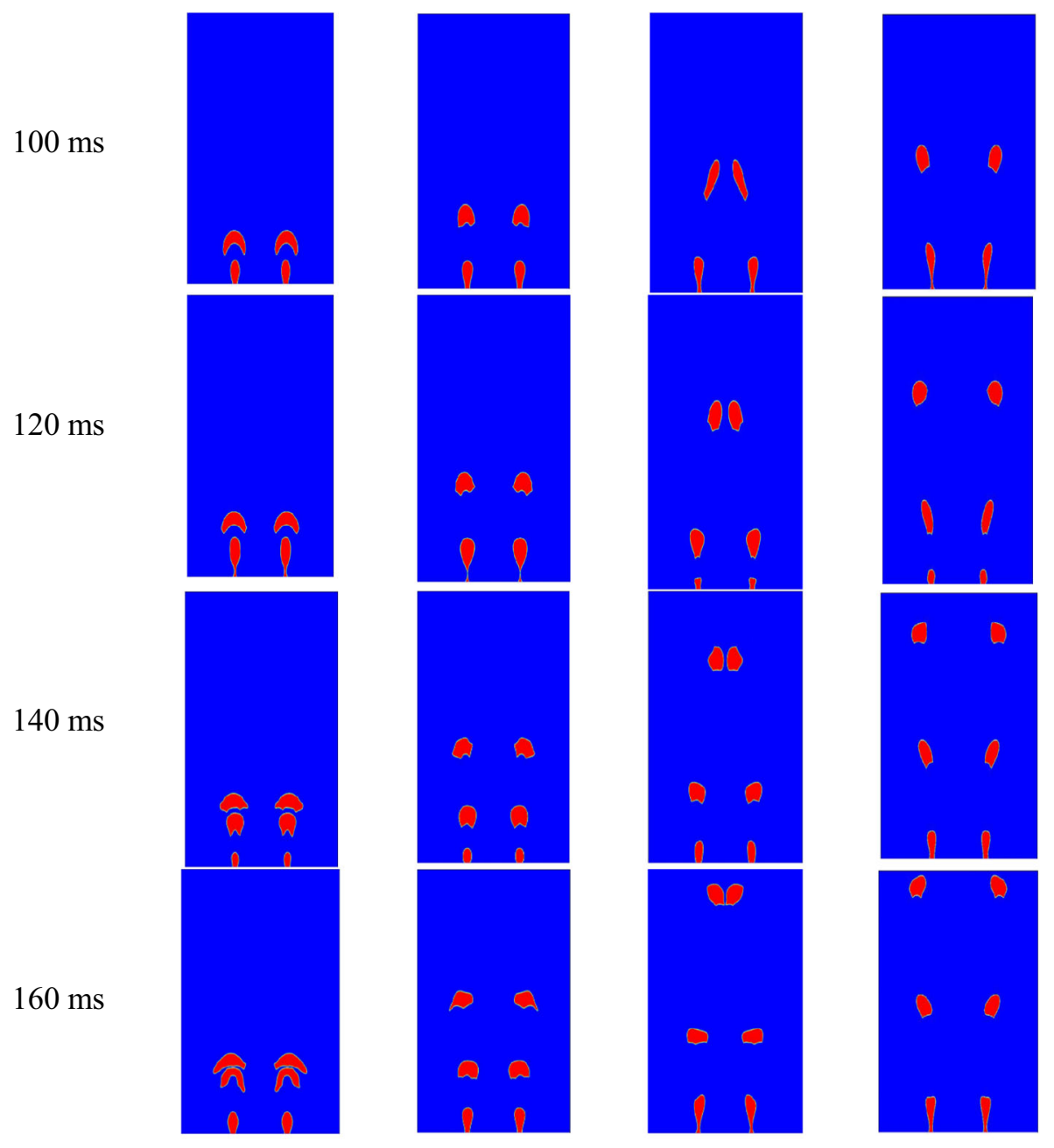

Figure 6. continued

equal slope of all the lines (with different $V_{r}$ values) as plotted in figure 6 . This is due to the fact that the gas inflow rate is constant throughout the simulation. The nature of the graph also depicts that at time $t=0$, bubble volume is nonzero, which further ensures the initial condition that the bubble is hemispherical initially (of radius equals to the orifice radius). It is observed from figure 6 that in a quiescent liquid $\left(V_{r}=0\right)$, the bubble is nearly spherical during the bubble formation period especially at lower values of $V_{r}$. However, in a co-flowing liquid $\left(V_{r}>0\right)$, initially the bubbles are approximately spherical in shape but subsequently the equilibrium shape of the bubbles prior to its detachment becomes non-spherical. Figure 6 shown here indicates that $V_{r}$ greatly influences the bubble detachment time (see table 2) and also the average bubble radius at detachment. Increasing $V_{r}$ brings about early detachment, resulting in a smaller bubble diameter due to the increase in the pressure forces acting on the surface of the bubble
Table 2. Effect of $V_{r}$ on bubble detachment time.

\begin{tabular}{llllll}
\hline Liquid to gas mean velocity ratio $V_{r}$ & 0 & 0.44 & 0.88 & 1.32
\end{tabular}

$\begin{array}{lllll}\text { Detachment time } t(\mathrm{~ms}) & 66.5 & 58.2 & 57.0 & 53.8\end{array}$

caused by the liquid inertia. Table 2 shows that as $V_{r}$ is increased, bubble detachment time is decreased due to the increased drag effect of the surrounding liquid.

It can also be observed from figure 6 that the vertical distance between the two consecutive detached bubbles is minimum in the case of quiescent ambient liquid whereas it increases with the increase in $V_{r}$, which means the phenomenon of coalescence/merger (fusion of two or more bubbles together) is more prone in the case of quiescent ambient condition. However, it is not so common in case of co-flowing condition because in this case previously detached bubble has risen sufficiently due to the dragging 
Table 3. Quantitative analysis of dynamic effect of co-flowing liquid on bubble formation.

\begin{tabular}{|c|c|c|c|c|c|}
\hline \multirow{2}{*}{\multicolumn{2}{|c|}{ Time $t(\mathrm{~ms})$}} & \multicolumn{4}{|c|}{ Bubble volume $\left(\mathrm{mm}^{3}\right)$} \\
\hline & & \multirow{2}{*}{$\frac{V_{b} \text { at } V_{r}=0}{3.6745}$} & \multirow{2}{*}{$\frac{V_{b} \text { at } V_{r}=0.44}{03.6745}$} & \multirow{2}{*}{$\frac{V_{b} \text { at } V_{r}=0.88}{03.6745}$} & \multirow{2}{*}{$\begin{array}{c}V_{b} \text { at } V_{r}=1.32 \\
03.6745\end{array}$} \\
\hline 0 & Single & & & & \\
\hline 10 & Single & 12.9513 & 12.9072 & 12.9072 & 12.9072 \\
\hline 20 & Single & 22.2097 & 22.2097 & 22.2097 & 22.2097 \\
\hline 30 & Single & 31.5122 & 31.5122 & 31.5122 & 31.5122 \\
\hline 40 & Single & 40.8147 & 40.8147 & 40.8147 & 40.8147 \\
\hline 50 & Single & 50.1172 & 50.0958 & 50.1172 & 50.1172 \\
\hline \multirow[t]{2}{*}{60} & Detached & 59.4197 & 54.4809 & 53.1324 & 50.4624 \\
\hline & Evolving & (not detached) & 4.9008 & 5.7492 & 8.9195 \\
\hline \multirow[t]{2}{*}{70} & Detached & 62.0983 & 54.4809 & 53.1324 & 50.4624 \\
\hline & Evolving & 6.5699 & 14.1871 & 15.0357 & 18.2058 \\
\hline \multirow[t]{2}{*}{80} & Detached & 62.0983 & 54.4809 & 53.1324 & 50.4624 \\
\hline & Evolving & 15.8562 & 23.4733 & 24.3218 & 27.4921 \\
\hline \multirow[t]{2}{*}{90} & Detached & 62.0983 & 54.4809 & 53.1324 & 50.4624 \\
\hline & Evolving & 25.1422 & 32.7593 & 33.6079 & 36.7785 \\
\hline \multirow[t]{2}{*}{100} & Detached & 62.0983 & 54.4809 & 53.1324 & 50.4624 \\
\hline & Evolving & 34.4284 & 42.0454 & 42.8939 & 46.0653 \\
\hline \multirow[t]{2}{*}{110} & Detached & 62.0983 & 54.4809 & 53.1324 & 50.4624 \\
\hline & Evolving & 43.8643 & 51.3316 & 52.1801 & 3rd bubble formed \\
\hline \multirow{2}{*}{120} & Detached & 62.0983 & 54.4809 & 53.1324 & 50.4624 \\
\hline & Evolving & 53.0014 & 60.6176 & 3rd bubble formed & 3rd bubble formed \\
\hline
\end{tabular}

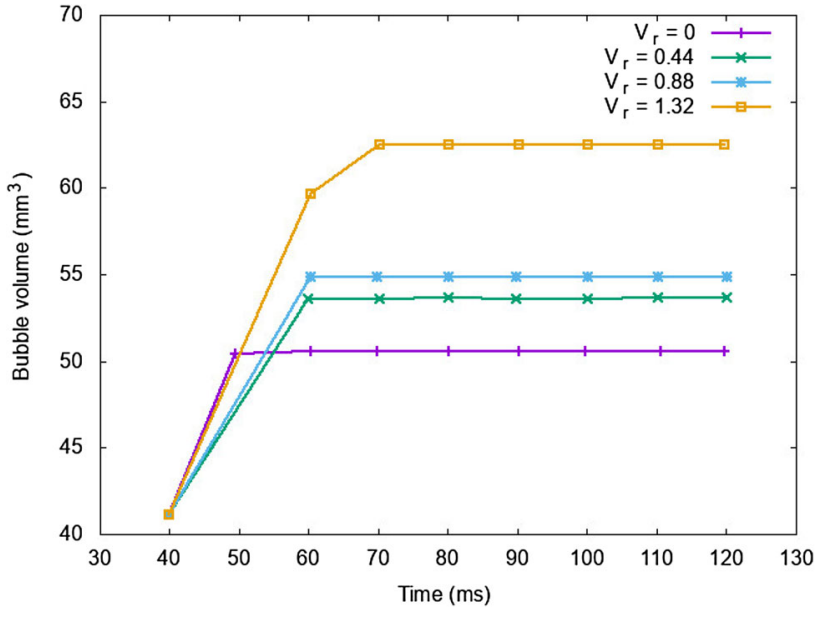

Figure 7. Average bubble size for different $V_{r}$ at given $W e=$ 4.35, $R e=690.48$ and $B o=0.31$.

effect of the surrounding liquid. Besides this, in case of $V_{r}=0.88$, two adjacent bubbles tend to converge towards the axis of the tank as they attend higher altitude, whereas in case of $V_{r}=1.32$, two adjacent bubbles try to diverge from the axis of the tank as they attain higher altitude. Though it seemed like the two adjacent bubbles in case of $V_{r}=0.88$ would interact with each other sidewise, it did not occur actually.

It is also interesting to note from figure 6 that at $t=120 \mathrm{~ms}$ for $V_{r}=0$ and 0.44 , the second bubbles are about to detach (with higher vertical distance travelled by

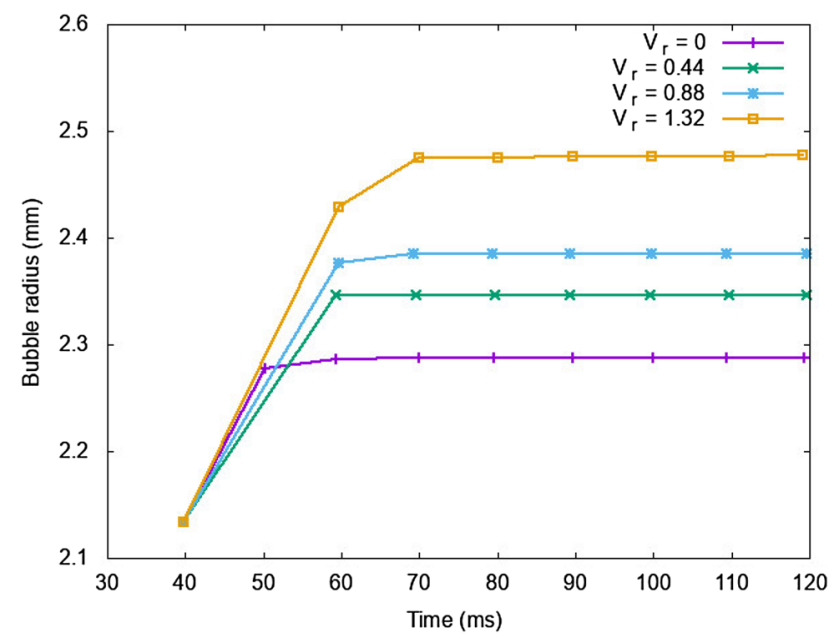

Figure 8. Average bubble radius for different $V_{r}$ at given $W e=4.35, R e=690.48$ and $B o=0.31$.

the first bubble for $V_{r}=0.44$ ) whereas for $V_{r}=0.88$ and 1.32 a third set of bubbles has started forming at the orifice (with higher altitude attained by the first and the second bubbles for $V_{r}=1.32$ ).

Table 3 presents a quantitative analysis of bubble volume at various time instants for the quiescent and the coflowing liquid ambience at $W e=4.35, R e=690.48$ and $B o=0.31$ and thereby reflects the dynamic effect of the coflowing liquid on the bubble formation. The volume calculation of bubble is performed in a $2 \mathrm{D}$ plane. The volume is calculated in the following simple steps: 


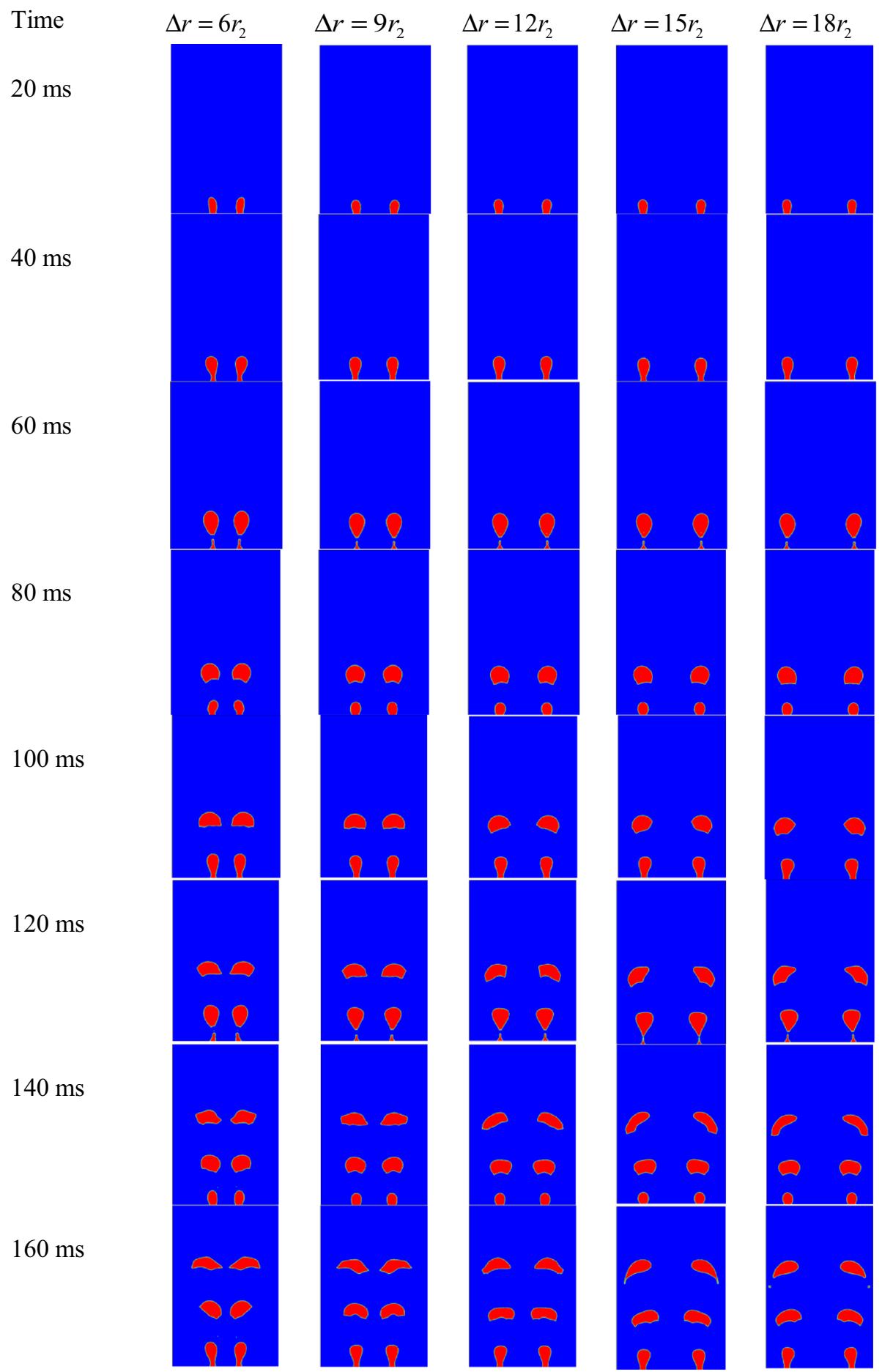

Figure 9. Effect of orifice spacing on bubbling synchronicity and frequency at given $V_{r}=0.44, W e=4.35, R e=690.48$ and $B o=0.31$.

(a) Total volume fraction of the gas phase is calculated at required time step for the selected region (which either contains an evolving bubble or a detached bubble as the case may be) of the computational domain by summing up the volume fractions of the individual cells in that region. (b) Now, since grids are uniform in nature, total volume of gas in the selected region is calculated by multiplying the total volume fraction of the gas phase in that region with the cell volume (i.e., cell area in 2D). This total volume of gas phase is nothing but the volume of the bubble residing in the selected region. 


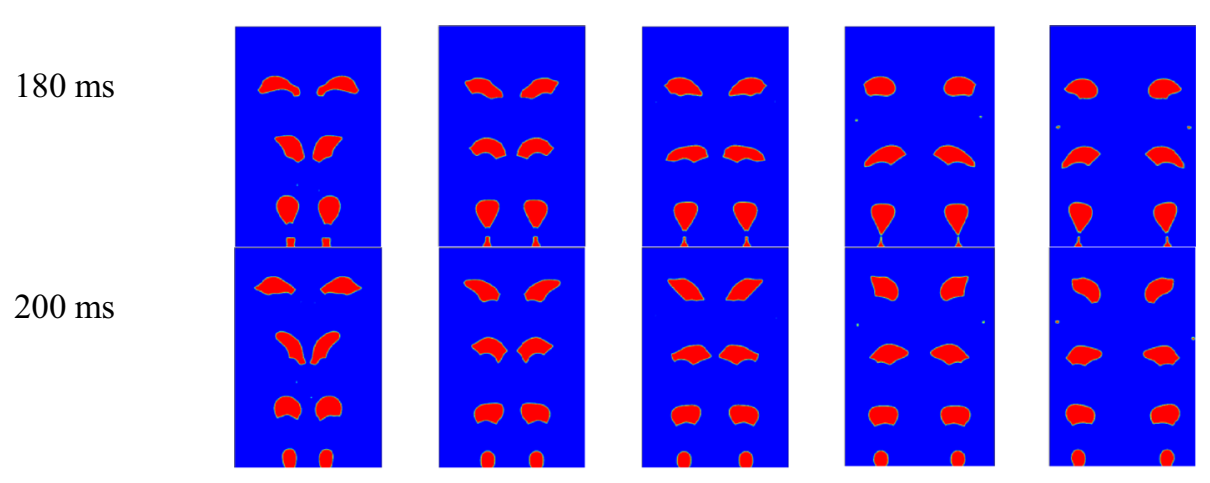

Figure 9. continued

(c) The intended region is selected by specifying four points (which encloses detached or evolving bubble) into a computer program developed for calculating the total volume fraction of the gas phase.

From table 3 we observe that the bubble detaches earlier in case of co-flowing (between 50 and $60 \mathrm{~ms}$ ) than in the case of quiescent ambient (between 60 and $70 \mathrm{~ms}$ ). With the help of data given in table 3 , bubble volume and average bubble radius are calculated using $r_{a v g}=\left(\frac{3 V_{b}}{4 \pi}\right)^{1 / 3}$ where $V_{b}$ is the bubble volume, and plotted in figures 7 and 8 , respectively. It can be concluded from figures 7 and 8 that the smallest size bubble is formed in case of $V_{r}=1.32$ whereas the largest size bubble is formed in case of $V_{r}=0$. After the bubble detaches from the orifice, its volume becomes constant and hence the graph becomes asymptotic and a new bubble starts forming at the tip of the orifice.

To understand the effect of orifice spacing on the bubbling synchronicity and frequency, several cases of different orifice spacings (such as three times, six times, nine times, twelve times, fifteen times and eighteen times of orifice radius) are run keeping all other parameters constant. If the orifice spacing is decreased below "four times of the orifice radius", side-by-side interaction between the two adjacent bubbles is observed.

Figure 9 reflects a very significant result that if we move from left to right in a particular row (which means an actual increase in the orifice spacing at a particular instant of time), the size of the bubbles in different cases remains the same. However, the orientation of the bubbles in case of larger orifice spacing goes on changing considerably as compared with that in smaller orifice spacing at the expense of time, particularly at 100 and $120 \mathrm{~ms}$. As we see, at time instants of 120 and $180 \mathrm{~ms}$, bubbles in larger orifice spacing are about to detach or just detached whereas in case of smaller orifice spacing it has risen appreciably. Thus, the bubble frequency is somewhat lowered in case of larger orifice spacing as compared with the smaller one but there is no significant effect on the bubbling synchronicity. However, if the orifice spacing is even below six times the orifice radius then bubbling synchronicity would be affected because the bubble formation would not be in the same phase anymore. Lastly, it has also been observed that the effect of orifice spacing at lower time instants (up to $80 \mathrm{~ms}$ ) is almost negligible but its effect at higher time instants are pretty much visible in terms of bubbles shape deviations and their orientations. The main purpose of considering so many orifice spacing values is to have better understanding on the effect of orifice spacing and to design a bubble generating device with optimized performance, minimum overall dimension and finally to meet the requirement in the least possible cost.

\section{Conclusions}

In this work two-dimensional numerical simulations are carried out to analyse the dynamics of gas-bubble formation from two submerged orifices in an immiscible Newtonian liquid under the condition of constant gas inflow rate using a finite-volume-based CFD solver Ansys Fluent. The study demonstrates the differences between the dynamic effects of the quiescent and the co-flowing liquid ambience on the bubble evolution and detachment processes. Some important results obtained in this study can be summarized as follows:

- Larger velocity of co-flowing liquid leads to faster bubble collapse dynamics and creates smaller size bubbles with enhanced bubble formation frequency and reduced bubble formation time. Thus, $V_{r}$ controls bubble size and bubbling time.

- With increasing $V_{r}$, the mean distance between the two consecutively detached bubbles increases, which suggests that chances of bubble coalescence is minimal at higher values of $V_{r}$. 
- Bubbling frequency in case of larger orifice spacing is somewhat lowered as compared with the smaller orifice spacing.

All in all, the study may lead to a pivotal role in controlling the size and the coalescence of the bubbles generated by appropriately selecting the co-flowing properties.

\section{List of symbols}

Bo Bond number

$C \quad$ Courant number

$\vec{g} \quad$ acceleration due to gravity

$H_{t} \quad$ height of the cylindrical tube

$\vec{n} \quad$ unit normal vector at the interface

$P \quad$ pressure

$P_{0} \quad$ atmospheric pressure

$r$ radial co-ordinate

$r_{2} \quad$ orifice radius

$R_{3} \quad$ distance of wall from central axis

Re Reynolds number

$Q_{g} \quad$ dispersed phase (gas) flow rate

$t \quad$ time

$u \quad$ velocity component in $r$-direction

$v \quad$ velocity component in $z$-direction

$v_{c, \text { avg }}$ liquid mean velocity at the inlet

$v_{\text {d.avg }}$ gas mean velocity at orifice inlet

$\vec{V} \quad$ velocity vector

$V_{b} \quad$ bubble volume

$V_{r} \quad$ liquid to gas mean velocity ratio

We Weber number

$z \quad$ axial co-ordinate

\section{Greek letters}

$\alpha \quad$ void fraction of liquid in two- phase cell

$\bar{\alpha} \quad$ smoothed void fraction field

$\delta_{s} \quad$ interface delta function

$\varepsilon \quad$ interface numerical thickness

$\kappa$ mean curvature of the interface

$\mu \quad$ dynamic viscosity of fluid

$\theta \quad$ azimuthal angle

$\rho$ density of fluid

$\sigma \quad$ surface tension coefficient

$\phi \quad$ level set function

$\phi_{s}$ static contact angle

\section{Subscripts}

avg average value

$c$ continuous phase (liquid)

$d$ disperse phase (gas)

$g \quad$ gas

$l \quad$ liquid

min minimum

$t$ tube

\section{Superscripts}

* dimensionless quantity

\section{References}

[1] Badam V K, Buwa V and Durst F 2007 Experimental investigations of regimes of bubble formation on submerged orifices under constant flow condition. Can. J. Chem. Eng. 85: $257-267$

[2] Majumdar S and Chowdhury S 2010 Novel therapeutic application of microbubbles for targeted drug delivery. Int. J. Pharm. Biol. Sci. 1: 1-9

[3] Marshall S H 1990 Air bubble formation from an orifice with liquid cross-flow. PhD Thesis, Department of Chemical Engineering, University of Sydney

[4] Kulkarni A A and Joshi J B 2005 Bubble formation and bubble rise velocity in gas-liquid systems: a review. Ind. Eng. Chem. Res. 44: 5873-5931

[5] Ranade V V 2002 Computational flow modeling for chemical reactor engineering, vol. 5. St. Louis, MO, USA: Academic Press

[6] Zaleski S 2005 Interface tracking-VOF. In: Lecture given at course: Industrial two-phase flow CFD. Belgium: von Karman Institute for Fluid Dynamics

[7] Huang M, Wu L and Chen B 2012 A piecewise linear interface-capturing volume-of-fluid method based on unstructured grids. Numer. Heat Transfer B 61: 412-437

[8] Davidson J F and Schüler B O G 1960 Bubble formation at an orifice in a viscous liquid. Trans. Inst. Chem. Eng. 38: 144-154

[9] Davidson J F and Schüler B O G 1960 Bubble formation at an orifice in an inviscid liquid. Trans. Inst. Chem. Eng. 38: 335-342

[10] Walters J and Davidson J 1963 The initial motion of a gas bubble formed in an inviscid liquid. J. Fluid Mech. 17: 321-336

[11] Kumar R and Kuloor N R 1970 The formation of bubbles and drops. Adv. Chem. Eng. 8: 255-368

[12] Longuet-Higgins M S, Kerman B R and Lunde K 1991 The release of air bubbles from an underwater nozzle. J. Fluid Mech. 230: 365-390

[13] Jamialahmadi M, Zehtaban M R, Müller-Steinhagen H, Sarrafi A and Smith J M 2001 Study of bubble formation under constant flow conditions. Chem. Eng. Res. Des. 79: 523-532

[14] Kasimsetty S S K, Manglik R M and Jog M A 2008 Computational modeling of dynamics of single bubbles emanating from capillary-tube orifice in an isothermal liquid pool. In: Proceedings of ASME IMECE, Boston, MA, October 31November 6, Paper No. IMECE2008-69251

[15] Chuang S C and Goldschmidt V W 1970 Bubble formation due to a submerged capillary tube in quiescent and coflowing systems. Trans. ASME J. Basic Eng. 92: 705-711

[16] Sada E, Yasunishi A, Katoh S and Nishioka M 1978 Bubble formation in flowing liquids. Can. J. Chem. Eng. 56: 669-672

[17] Gerlach D, Alleborn N, Buwa V and Durst F 2007 Numerical simulation of periodic bubble formation at a submerged orifice with constant gas flow rate. Chem. Eng. Sci. 62: 2109-2125

[18] Sussman M and Puckett E G 2000 A coupled level set and volume-of-fluid method for computing $3 \mathrm{D}$ and axisymmetric incompressible two-phase flows. J. Comput. Phys. 162: 301-337 
[19] Sevilla A, Gordillo J M and Martinez-Bazan C 2005 Bubble formation in a co-flowing air-water stream. J. Fluid Mech. 530: $181-195$

[20] Terasaka K, Tsuge H and Matsue H 1999 Bubble formation in concurrently upward flowing liquid. Can. J. Chem. Eng. 77: 458-464

[21] Gordillo J M, Sevilla A and Martinez-Bazan C 2007 Bubbling in a co-flow at high Reynolds numbers. Phys. Fluids 19: 077102-01-18

[22] Chakraborty I, Biswas G and Ghoshdastidar P S 2011 Bubble generation in quiescent and co-flowing liquids. Int. J. Heat Mass Transfer 54: 4673-4688

[23] Zuiderweg F J 1982 Sieve trays-a view on the state of the art. Chem. Eng. Sci. 37: 1441-1461

[24] Wijn E F 1998 On the lower operating range of sieve and valve trays. Chem. Eng. J. 70: 143-155

[25] Miyahara T, Matsuba Y and Takahashi T 1983 The size of bubbles generated from perforated plates. Int. J. Chem. Eng. 23: 517-602

[26] Titomanlio G, Rizzo G and Acierno D 1976 Gas bubble formation from submerged orifices "simultaneous bubbling" from two orifices. Chem. Eng. Sci. 31: 403-404

[27] Ruzicka M, Drahos J, Zahradnik J and Thomas N H 1999 Natural modes of multi-orifice bubbling from a common plenum. Chem. Eng. Sci. 54: 5223-5229
[28] Ruzicka M, Drahos J, Zahradnik J and Thomas N H 2000 Structure of gas pressure signal at two-orifice bubbling from a common plenum. Chem. Eng. Sci. 55: 421-429

[29] Xie S and Reginald B H T 2003 Bubble formation at multiple orifices-bubbling synchronicity and frequency. Chem. Eng. Sci. 58: 4639-4647

[30] Pereira Dias M I 1999 Bubble formation at multiple orifice plate submerged in quiescent liquid. $\mathrm{PhD}$ Thesis, University Libre De Bruxelle and Von Karman Institute for Fluid Dynamics

[31] Brackbill J U, Kothe D B and Zemach C A 1992 Continuum method for modeling surface tension. J. Comput. Phys. 100: 335-354

[32] Hirt C W and Nichols B D 1981 Volume of fluid (VOF) method for the dynamics of free boundaries. J. Comput. Phys. 39: 201-225

[33] Ansys Fluent ver. 14.0 2011 Theory guide. Canonsburg, USA: Ansys Inc.

[34] Leonard B P 1979 A stable and accurate convective modelling procedure based on quadratic upstream interpolation. Comput. Methods Appl. Mech. Eng. 19: 59-98

[35] Patankar S V 1980 Numerical heat transfer and fluid flow. Washington, DC: Hemisphere

[36] Courant R, Friedrichs K and Lewy H 1928 Über die Partiellen Differenzengleichungen der Mathematischen Physik. Math. Ann. 100: 32-74 